LA-UR-, $\quad 98-3626$

\title{
Title: A HIGH-DAMAGE-THRESHOLD PINHOLE FOR GLASS FUSION LASER APPLICATIONS
}

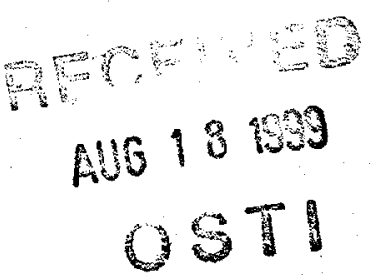

Author(s): N. A. Kurnit, P-24

S. A. Letzring, P-24

R. P. Johnson, P-24

Submitted to: THIRD ANNUAL INTERNATIONAL CONFERENCE ON SOLID STATE LASERS FOR APPLICATION TO INTERTIAL CONFINEMENT FUSION

MONTEREY, CA 7-12 JUNE 1998

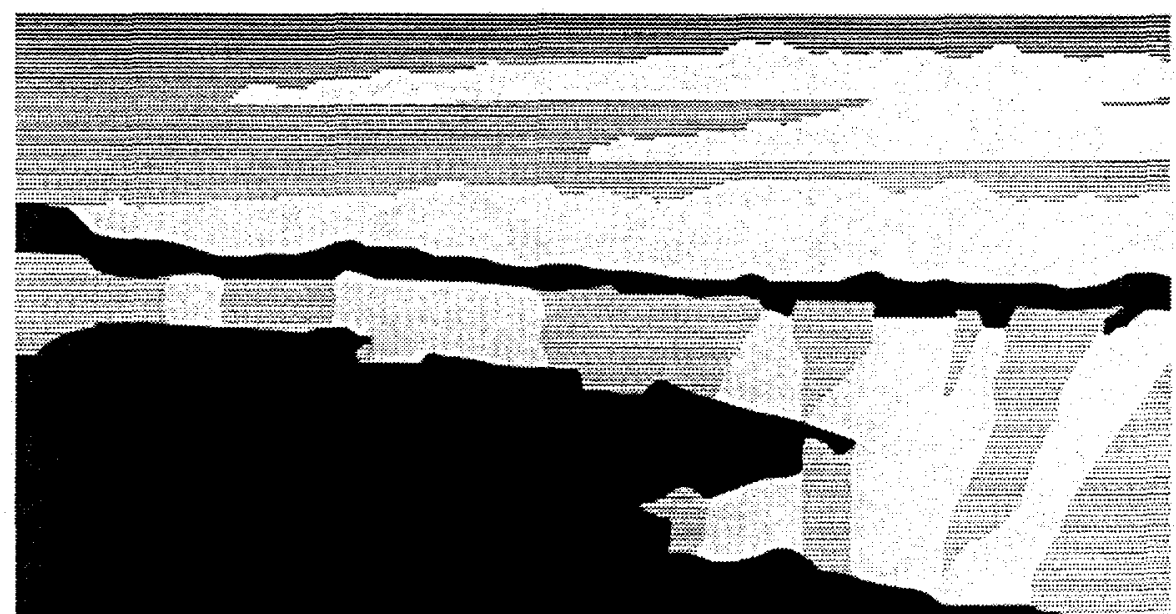

Los Alamos National Laboratory, an affirmative action/ equal opportunity employer, is operated by the University of California for the U.S. Department of Energy under contract W-7405-ENG-36. By acceptance of this article, the publisher recognizes the the U.S. Government retains a nonexclusive, royalty-free license to puplish or reporduce the published form of this contribution, or to allow others to do so, for U.S. Government purposes. The Los Alamos National Laboratory requests that the publisher identify this article as work performed under the auspices of the U.S. Department of energy. 


\section{DISCLAIMER}

This report was prepared as an account of work sponsored by an agency of the United States Government. Neither the United States Government nor any agency thereof, nor any of their employees, make any warranty, express or implied, or assumes any legal liability or responsibility for the accuracy, completeness, or usefulness of any information, apparatus, product, or process disclosed, or represents that its use would not infringe privately owned rights. Reference herein to any specific commercial product, process, or service by trade name, trademark, manufacturer, or otherwise does not necessarily constitute or imply its endorsement, recommendation, or favoring by the United States Government or any agency thereof. The views and opinions of authors expressed herein do not necessarily state or reflect those of the United States Government or any agency thereof. 


\section{DISCLAIMER}

Portions of this document may be illegible in electronic image products. Images are produced from the best available original document. 
A high-damage-threshold pinhole for glass fusion laser applications

N. A. Kurnit, S. A. Letzring and R. P. Johnson

Group P-24, MS E526

Los Alamos National Laboratory

Los Alamos, NM 87545

\begin{abstract}
We are investigating methods to fabricate high-damage-threshold spatial-filter pinholes that might not be susceptible to plasma closure for relatively high energies and long pulses. These are based on the observation that grazing-incidence reflection from glass can withstand in excess of $5 \mathrm{~kJ} / \mathrm{cm}^{2}$ (normal to the beam) without plasma formation. The high damage threshold results from both the $\cos \theta$ spreading of the energy across the surface and the reflection of a large fraction of the energy from the surface, thereby greatly reducing the field strength within the medium.
\end{abstract}

Keywords: Spatial filters, optical breakdown, damage thresholds

\title{
1. INTRODUCTION
}

An important component in glass laser fusion sytems are the far-field apertures (spatial-filter "pinholes") that are needed in order to limit the growth of beam perturbations arising from propagation of high intensity beams through long pathlengths of glass having a substantial nonlinear index. Unless these " $B$ integral" perturbations are removed by sufficiently small spatial filter apertures, beam filamentation and optical breakdown can result in damage to downstream optics. The present method of pinhole fabrication being pursued for the National Ignition Facility (NIF) utilizes tapered conical metallic pinholes that reflect unwanted light in the wings of the beam out of the beam path either by the metallic surface or by the plasma formed on the metallic surface after breakdown threshold is reached.1,2 A limitation of these pinholes is that the plasma flows into the center of the pinhole and will result in beam refraction and eventually pinhole closure. Present results indicate that these pinholes are adequate for the level of spatial filtering required at NIF, ${ }^{3}$ but other methods would be desirable to achieve even tighter spatial filtering.

Our pinhole concept is similar to the one described in Ref. 2, except for the use of a smooth glass surface in place of the metallic surface, as well as some differences in the preferred pinhole geometry that result from the transmission of energy into the glass surface. Potential advantages of the glass pinhole are that the breakdown threshold on grazing-incidence glass surfaces can be very high, leading to the possibility of not creating a plasma that can lead to pinhole closure, and that modifications of presently available commercial techniques for reproducibly making tapered capillaries with inner surfaces produced directly from molten material ${ }^{4}$ could lead to relatively low fabrication costs. We first describe some damage threshold measurements made in support of this concept, and then discuss design options and initial attempts at fabrication and testing of such glass pinholes.

\section{DAMAGE THRESHOLD MEASUREMENTS}

Measurements of the angular dependence of damage thresholds on absorbing glass have recently been reported, ${ }^{5}$ but are discussed here in greater detail. The reasons for the choice of absorbing glass for these measurements were two-fold. First of all, the damage threshold for absorbing glass tends to be more deterministic than that of transparent glass, as long as obvious surface imperfections are avoided, thus making the measurements more reproducible. Secondly, the damage for non-absorbing flat glass samples almost always occurs at considerably lower levels on the exit surface of the glass, particularly for angles near grazing incidence, and it occurs catastrophically, working its way back toward the front surface on subsequent shots, thereby often making determination of the front surface damage threshold difficult. This is well understood in terms of the addition of the reflected field amplitude to that of the incident field on the rear surface, ${ }^{6}$ leading at normal incidence to a 1.44-fold increase in the square of the local field strength on the exit surface (for $\mathrm{n}=1.5$ ), and a nearly 4-fold increase (for s-polarized light, 2 -fold for p-polarized light) near grazing incidence where the field is almost totally reflected. The breakdown usually starts on the surface due to further enhancement of the field strength by surface imperfections, ${ }^{7}$ but the plasma created by the breakdown reflects sufficient energy to produce enhanced field strengths further back in the sample, leading to bulk damage extending back toward the source. This rear-surface breakdown can be avoided by using an absorbing glass with sufficient absorption to attenuate the beam below breakdown level on the rear surface. At the 
same time, the absorption should not be so strong as to greatly lower the damage threshold on the front surface. Higher thresholds should in principle be obtainable with nonabsorbing glass provided the rear surface problems can be avoided, and we will discuss below some techniques for achieving this.

The measurements reported here were made on Schott NG-4 neutral density filter glass, which was found to have a relatively-high front-surface damage threshold and sufficiently low transmission $(0.3 / \mathrm{mm})$ to not give rear surface breakdown for a 3-mm thick sample. In fact, the measured near-normal incidence threshold of $60-80 \mathrm{~J} / \mathrm{cm}^{2}$ for a 4.5-5 ns FWHM pulse is sufficiently high as to raise some questions as to the accuracy of these measurements. Earlier measurements had given 14 $\mathrm{J} / \mathrm{cm}^{2}$ for this type of glass for a 3-ns pulse. ${ }^{8}$ However, there may be a number of reasons for this discrepancy: our slightly longer pulse length, the use of a temporally smooth, injection-seeded single-longitudinal-mode laser, improvements in polishing technology since these earlier measurements, the fact that our measurements were performed in vacuum rather than air, and the use of a much smaller spot in our measurements, leading to a different definition of what we consider to be damage threshold. In fact, we purposely used a collinear He-Ne beam to avoid regions of the sample that exhibited scattering before irradiation. Our measured damage threshold should thus be taken to be representative of the intrinsic threshold of the material, only slightly influenced by the presence of surface imperfections. We consider this to be of more interest for the purpose at hand, because the techniques we envision for construction of pinholes result in laser-melted surfaces which should be free of scratches and inclusions, and which have been shown in the past to give damage thresholds substantially enhanced from mechanically polished surfaces. ${ }^{9}$ It is also possible that some of the fabrication procedures for colored glass filters have changed since the measurements in Ref. 8, because the filters that appeared most promising from those measurements, UG-5 and UG-11, did not perform as well as NG-4 in our measurements.

The measured damage threshold 5 as a function of angle from grazing is given in Fig. 1. As can be seen, the damage threshold increases by about two orders of magnitude by going to grazing angles smaller than $2^{\circ}$. The theoretical curves give the calculated damage threshold, normalized to the damage threshold at normal incidence, assuming that damage will occur when the square of the electric field strength within the medium exceeds some value characteristic of the medium, where the internal electric field strength is calculated from the Fresnel relations, ${ }^{10}$ as

$$
t_{p}=\frac{2 \sin \theta_{t} \cos \theta_{i}}{\sin \left(\theta_{i}+\theta_{t}\right) \cos \left(\theta_{i}-\theta_{t}\right)} a_{p}
$$

for the input field amplitude $a_{p}$ polarized parallel to the plane of incidence, and

$$
\mathrm{t}_{\mathrm{s}}=\frac{2 \sin \theta_{\mathrm{t}} \cos \theta_{\mathrm{i}}}{\sin \left(\theta_{\mathrm{i}}+\theta_{\mathrm{t}}\right)} \mathrm{a}_{\mathrm{s}}
$$

for the input field amplitude a polarized perpendicular to the plane of incidence, where $\theta_{\mathrm{i}}$ is the angle of incidence (measured from the surface normal), and $\theta_{t}$ is the angle of the refracted beam. Most of the data was taken for the lower-damagethreshold p-polarization, although a few points are shown also for s-polarization. No damage was observed at the maximum power available for the points marked by arrows at $2^{\circ}$ for s-polarization and $0.9^{\circ}$ for p-polarization, although other spots damaged at close to this fluence. No spots damaged at this maximum level for s-polarization at $0.9^{\circ}$. It is seen that damage thresholds in excess of $5 \mathrm{~kJ} / \mathrm{cm}^{2}$ (in the normal-incidence beam) are obtained for grazing angles of less than $2^{\circ}$, corresponding to peak intensities of $>10^{12} \mathrm{~W} / \mathrm{cm}^{2}$. This is believed to be in the range of interest for spatial filter pinholes for the NIF. Non-absorbing glass can almost certainly exhibit even higher thresholds. A few measurements done at grazing incidence on stainless steel and copper mirror surfaces indicated plasma thresholds at approximately 100 and 50 times lower fluences, respectively.

Data was taken by exposing the sample to several shots at fixed fluence on the same spot, and then increasing the fluence by rotating a half-wave plate between crossed polarizers and repeating until a plasma plume was consistently observed on the surface. Often, a plasma was observed at much lower levels and then cleaned up due to removal of contaminants; this was ignored unless it grew to become a consistent plasma. Some increased scattering of a He-Ne beam on the irradiated spot could sometimes be seen just prior to the shot on which plasma breakdown occurred. The damaged surface generally showed a roughened appearance, but without extensive pitting or material removal. As can be seen from the data, reproducibility of the threshold from spot to spot was typically within $20-25 \%$. The fact that the small-angle data tends to fall somewhat below the curves may result from the higher probability of hitting an imperfection for the larger beam footprint near grazing incidence. The fluence of the normal-incidence beam was calibrated by taking a CCD image of 


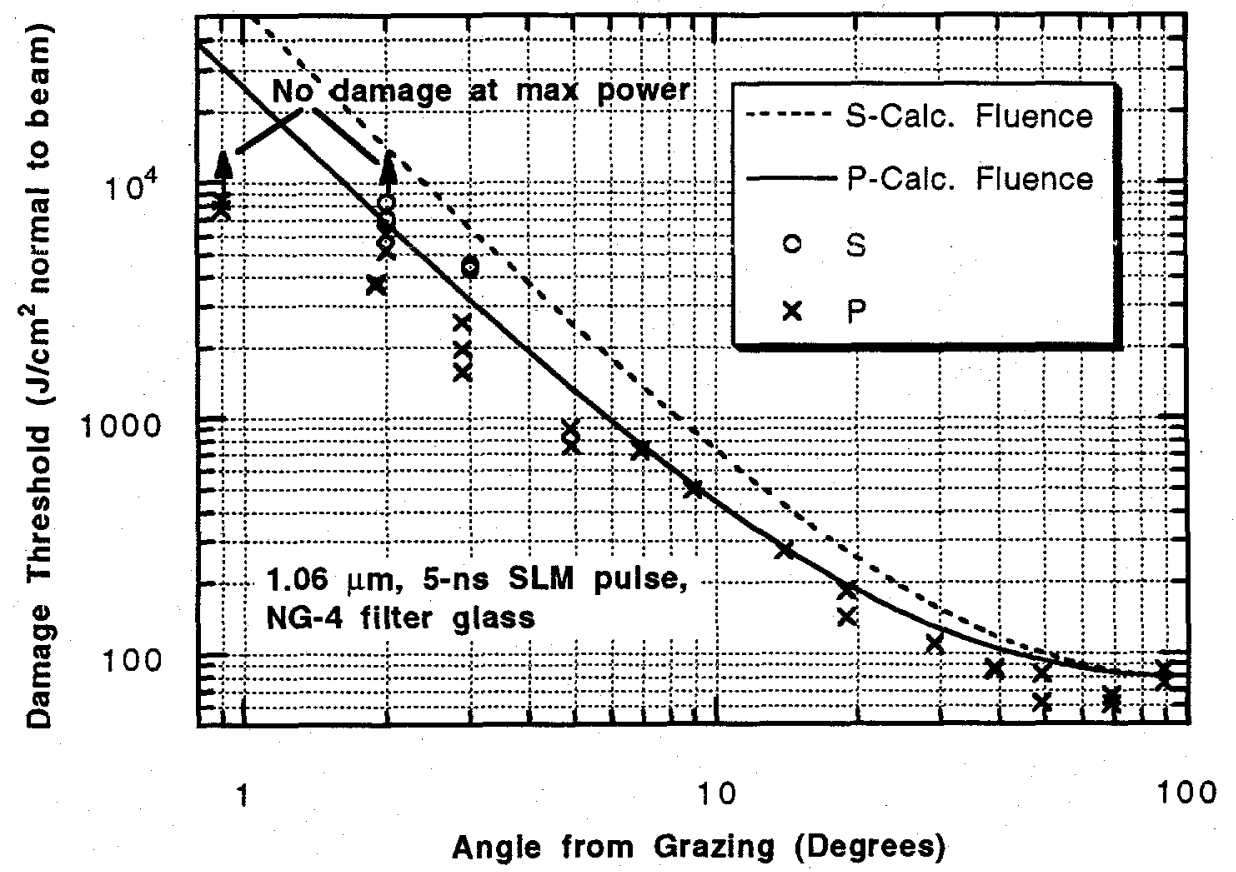

Fig. 1. Experimental damage threshold on NG-4 filter glass compared to theoretical expectation for angular dependence (normalized to normal-incidence damage threshold).

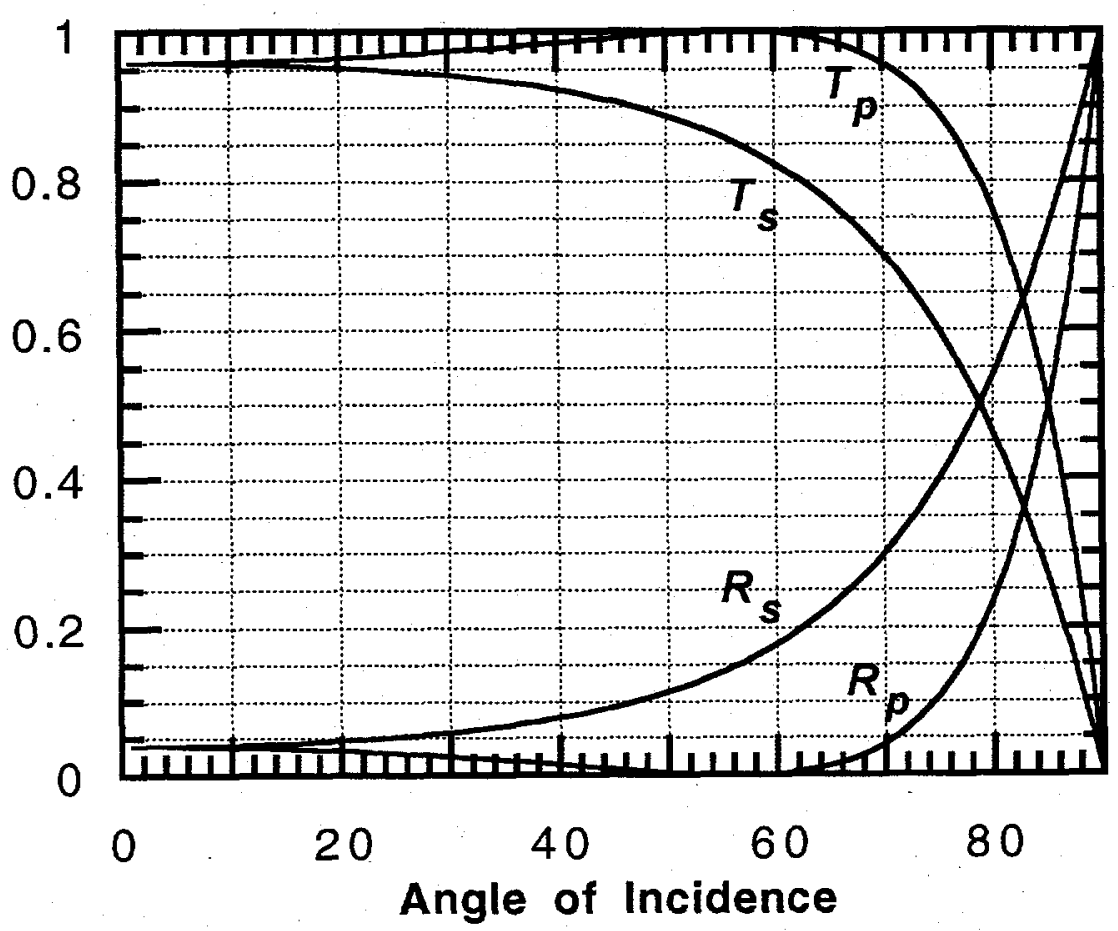

Fig. 2. Reflection and transmission coefficients for $n=1.5$ glass for $\mathrm{s}$ - and $\mathrm{p}$-polarized radiation as a function of angle from surface normal. 
the focused beam at an equivalent focal spot off an uncoated surface and with the half-wave plate turned to minimum transmission and additional attenuation done by means of reflective mirrors placed before the focusing lens. The measured spot size was about $70 \mu \mathrm{m}$ FWHM, but because the wings of the beam contained more energy than a gaussian, the peak fluence was calculated by integrating the area under the beam profile, and corresponded to an effective beam area of very nearly $10^{-4} \mathrm{~cm}^{2}$. It is believed that the calibration is accurate to within $\pm 20 \%$, but relative measurement accuracy is limited primarily by the statistics of the damage process.

The increase in damage threshold for small grazing angles can be viewed as being due to two factors: the $\cos \theta_{\tau} / \cos \theta_{2}$ increase in cross section of the transmitted beam relative to the incident beam, and the reflection of a large fraction of the incident beam from the surface. This last factor is shown in Fig. 2 for $n=1.5$ glass. The transmittance and reflectance of the beam are related by:

$$
T=\frac{\mathrm{n} \cos \theta_{\mathrm{t}}|\mathrm{t}|^{2}}{\cos \theta_{\mathrm{i}}|\mathrm{a}|^{2}}=1-R \text {. }
$$

The fraction of energy penetrating into the surface falls very rapidly below $4^{\circ}$ from grazing, dropping at $2^{\circ}$ to about $10 \%$ for s-polarized light and $20 \%$ for p-polarized light. The $\cos \theta_{\imath}$ factor is also dropping linearly with the grazing angle, $90-\theta_{\imath}$ in this region; these two factors combine to give the large increase in predicted threshold shown in Fig.1.

\section{PINHOLE DESIGN CONSIDERATIONS}

In designing a glass pinhole, it is important to consider whether the energy transmitted into the glass can cause damage somewhere. This is less of a problem for absorbing glass, but the highest-damage threshold pinholes should be attainable with an inner surface made of non-absorbing glass. A possible design is shown in Fig. 3. The input taper is made at a 3-4 full angle so as to expand slightly faster than the $\mathrm{f \#}$ of the beam. In the case shown, the inner region is made of nonabsorbing glass, and a cladding of absorbing material is bonded to the outside. Ideally, this would be absorbing glass if a suitable match of expansion coefficients can be found such that a non-reflective fusion bond can be achieved. Alternatively, it is possible to coat the glass with an index-matched polymer material; we have utilized UV-curable epoxy in initial attempts at construction of such pinholes. Because the beam cylindrically expands before reaching the epoxy material, this lower-damage-threshold material may withstand the lower field strengths that exist in this region. Although these lower field strengths might also not be sufficent to damage an uncladded boundary, it is important to eliminate the reflection at this surface that would refocus energy back onto the inner surface of the glass. Because this energy is incident going from high index to low index, the reflected field adds to the incident field on this boundary, as previously discussed, making it more damage prone than the spot on which the original beam is incident. The same is true of the beam reflected from the outside of the cladding if a cylindrical geometry is maintained, unless the cladding is loaded with an absorbing or scattering material, which is easily accomplished. Other methods that could be effective at avoiding strong refocusing of the beam on the inner surface are to alter the cylindrical geometry on the outer surface, as for example in the alternative shown where the surface is made more nearly square. Another possibility is to put a sawtooth corrugation or screw thread on the outer surface; in this case most of the energy incident on this surface passes through a near-normal-incidence surface (the internal angle of the beam is typically near $40^{\circ}$ ).

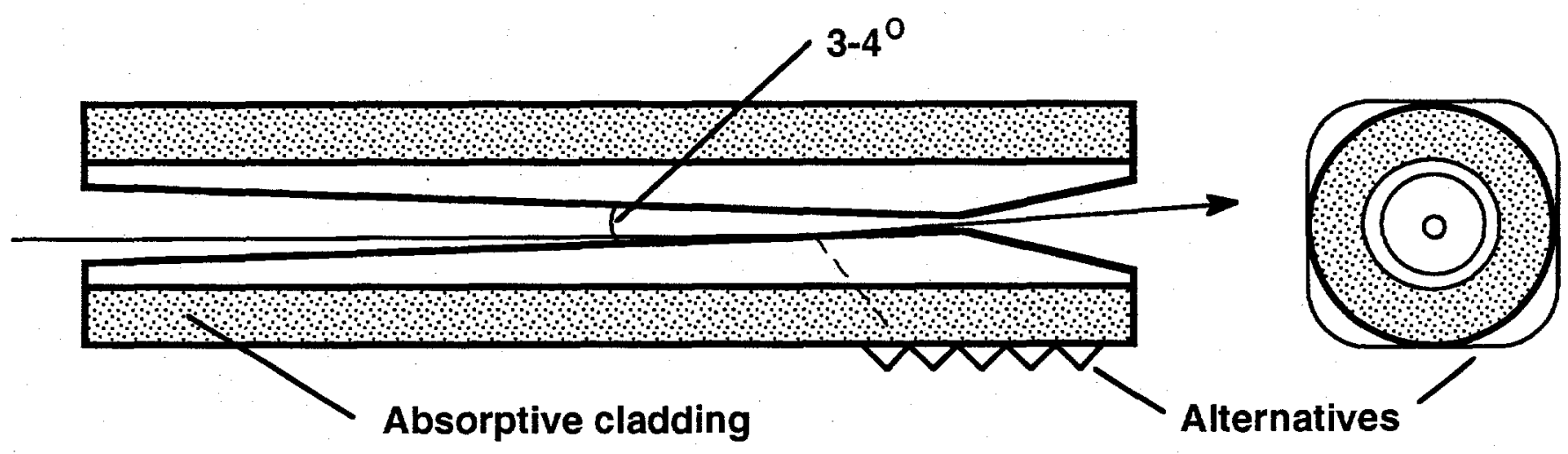

Fig. 3. Example pinhole design with alternative treatments to outer surface to prevent breakdown from reflected beams. 
Another feature of the glass pinhole that is somewhat different than the metal pinhole is that it is important to have an output cone as well as an input cone. Otherwise the energy refracted near the pinhole throat will be incident on the vertical wall of the pinhole and almost certainly cause damage on the output face. The output taper should have a large enough angle that none of the energy reflected on the pinhole surface will be redirected into the acceptance aperture of the beam. Another difference is that the metal pinholes are typically designed so that only one bounce is permitted before the beam exits through the pinhole, in order to avoid concentration of energy toward the pinhole throat. This is not as important in the case of the glass pinhole (as long as it remains below breakdown threshold) because the angle of the beam from grazing increases on each reflection, so that less of the energy is reflected downstream. As can be seen from Fig. 2, this is a fairly rapid function of the angle. This can also be used to advantage on the input taper if this taper is slightly flared into a horn shape. This gets the input face more quickly away from the low intensity edges of the beam that might otherwise cause breakdown on this surface. The damage threshold of the taper will be somewhat lower than near the throat, but the beam intensity is much lower here, and very little of this energy will survive downstream after a few low reflectivity bounces. As in the case of the metal pinholes, the outer leading edge of the pinhole can also be cut back at approximately $45^{\circ}$ to deflect a large fraction of any plasma created on the input face away from the opening.

\section{CONCLUSIONS}

We believe the glass pinholes described here provide a viable approach to construction of non-closing spatial filters for high-energy glass lasers. Some initial pinholes have been fabricated from somewhat smaller diameter tubes than a final design might utilize, and have been coated with UV-curable epoxy. These will be tested on the Trident laser facility at Los Alamos as scheduling permits.

\section{ACKNOWLEDGMENTS}

This work was performed under the auspices of the US Department of Energy as a joint collaborative effort between the Los Alamos National Laboratory under contract W-7405-ENG-36 and the Lawrence Livermore National Laboratory under contract W-7405-ENG-48, both operated by the University of California. We are grateful to Jim Murray and David Milam of LLNL for many helpful discussion, and to Robert Harrison of Los Alamos for his technical assistance in performing damage measurements.

\section{REFERENCES}

1. K. G. Estabrook, P. Celliers, J. Murray, R. Wallace, G. Stone, B. Van Wonterghem, B. MacGowan, J. Hunt, and K. Manes, "An improved pinhole spatial filter," 26th Annual Anomalous Absorption Conference, Fairbanks, Alaska, Aug. 26-30, 1996.

2. P. M. Celliers, K. G. Estabrook, R. J. Wallace, J. E. Murray, L. B. Da Silva, B. J. MacGowan, B. M. Van Wonterghem, and K. R. Manes, "Spatial filter pinhole for high-enery pulsed lasers," Appl. Opt. 37, 2371 (1998).

3. J. E. Murray, private communication.

4. For example, capillary gas chromatography unions from MicroQuartz Sciences, Phoenix, AZ.

5. N. A. Kurnit and R. F. Harrison, "Measurement of the angular dependence of damage thresholds of absorbing glass for application to high-damage-threshold far-field apertures," Conf. on Lasers and Electro-Optics (CLEO'98), San Francisco, CA, May 4-8, 1998, paper CTh035.

6. M. D. Crisp, N. L. Boling and G.Dubé, "Importance of Fresnel reflections in laser surface damage of transparent dielectrics," Appl. Phys. Letters 21, 364 (1972); N. L. Boling, M. D. Crisp, and G. Dubé, "Laser induced surface damage," Appl. Opt. 12, 650 (1973).

7. N. Bloembergen, "Role of cracks, pores, and absorbing inclusions on laser induced damage threshold at surfaces of transparent dielectrics," Appl. Opt. 12, 661 (1973).

8. H. Hack and N. Neuroth, "Resistance of optical and colored glasses to 3-ns laser pulses," Appl. Opt. 21, 3239 (1982).

9. P. A. Temple, D. Milam, and W. H. Lowdermilk, $\mathrm{CO}_{2}$-laser polishing of fused silica surfaces for increased laser damage resistance at $1.06 \mu \mathrm{m}, " 1979$ Boulder Laser Damage Symposium, NBS Spec. Pub. 568, p. 229.

10. See, for example, M. Born and E. Wolf, Principles of Optics, Sec. 1.5. 